\title{
GYNAECOLOGICAL PRESENTATION OF SYSTEMIC MALIGNANCIES
}

\section{Dr Swarnabindu Banerjee}

\section{INTRODUCTION:}

\section{ROUND CELL TUMOURS/HAEMATOLYMPHOID MALIGNANCIES}

- presenting with gynaecological symptoms are not infrequent

- often those are a component of symptom complex

- which have a clue towards the eitology.

ROUND CELL TUMOURS/HAEMATOLYMPHOID

MALIGNANCIES presenting solely as structural lesions of female genital tract are really uncommon. Here I would depict a series of 10 cases which were treated in the department of Medical Oncology, Medical College Kolkata, India between 2014 June to 2017 June.

\section{MATERIALS AND METHODS:}

Cases were selected from outpatient, indoor and day care records.

CT scan of (Thorax +Abdomen + Pelvis ) were done in all cases.

The diagnosis was established either by image guided biopsy or by laparotomy and subsequently by histopathology and Immunohistochemistry.

Epidemiological data age, type of malignancy, sites of involvement, method of diagnosis, chemotherapy drugs, duration of therapy, grade III/IV adverse effects, outcome were recorded. Number of cases were too small for any statistical analysis hence only descriptive statistics has been used here.

\section{RESULTS:}

Number of cases $=10$. Age range $=5 y$ rs-

45yrs. Median age $=25 \mathrm{yrs}$.

Median follow up=3.5 yrs.

Median duration of symptoms=3mons.

Symptoms were pain abdomen in 9 cases

abdominal distension in 4 cases,

fever in 1case,

incidental lump in abdomen detected by mother in

1 case..

\section{CONCLUSION:}

It is quite clear that haenatolympold malignancies outnumber others .Clue to suspicion of ROUND CELL TUMOURS/HAEMATOLYMPHOID MALIGNANCIES are younger age, prominence of adnexal mass regional lymphadenopathy and absence of ascites, mass lesion of uterus and adnexae without ascites and lymphadenopathy.Early diagnosis is necessary since the above group of diseases represent curable malignancies even at advanced stages so we need to treat before their performance score deteriorates.
Sites of involvement were

- tubes and ovaries in 9 cases,

- pelvic and retroperitoneal lympadenopathy in 8 cases,

- kidney in 1 case,

- ascites in 1 case, omental thickening in 1case,

- pleural effusion in 1 case.

The diagnosis was established by

- CT guided core biopsy from adnexal mass in 1 case, retroperitoneal lymph node in 1 case,

- immunophenotyping of ascitic fluid in 1 case,

- immunophenotyping of bone marrow in 1 case,

- rest by laparotomy

The diagnoses were

- Pre-B ALL in 1 case

- $\mathrm{ALCL}$ in 1 case

- paracervical Ewings Sarcoma in 1 case

- ovarian Ewings Sarcoma in 1 case

- Burkitts Lymphoma in 3 cases

- DLBCL in 2 cases

- high grade lymphoma Burkitts like in 1 case.

- The median latency from symptom onset to start of treatment was 1.5 months.

- The treatment was by multiagent noncrossresistant chemotherapy according to existing guidelines.

- 1 case of Burkitts Lymphoma and 1 case of DLBCL suffered from toxic death on treatment. 